\title{
Inhibition of ALK enzymatic activity in T-cell lymphoma cells induces apoptosis and suppresses proliferation and STAT3 phosphorylation independently of Jak3
}

\author{
Michal Marzec ${ }^{1}$, Monika Kasprzycka ${ }^{1,2}$, Andrzej Ptasznik ${ }^{3}$, Pawel Wlodarski ${ }^{1}$, Qian Zhang ${ }^{1}$, \\ Niels Odum ${ }^{4}$ and Mariusz A Wasik ${ }^{1}$ \\ ${ }^{1}$ Department of Pathology and Laboratory Medicine, University of Pennsylvania, Philadelphia, PA, USA; \\ ${ }^{2}$ Department of Clinical Immunology, Warsaw Medical University, Warsaw, Poland; ${ }^{3}$ Department of Medicine, \\ Division of Hematology and Oncology, University of Pennsylvania, Philadelphia, PA, USA and ${ }^{4}$ Institute \\ of Medical Microbiology and Immunology, University of Copenhagen, Copenhagen, Denmark
}

\begin{abstract}
Aberrant expression of the ALK tyrosine kinase as a chimeric protein with nucleophosmin (NPM) and other partners plays a key role in malignant cell transformation of T-lymphocytes and other cells. Here we report that two small-molecule, structurally related, quinazoline-type compounds, WHI-131 and WHI-154, directly inhibit enzymatic activity of NPM/ALK as demonstrated by in vitro kinase assays using a synthetic tyrosine-rich oligopeptide and the kinase itself as the substrates. The inhibition of NPM/ALK activity resulted in malignant T cells in suppression of their growth, induction of apoptosis and inhibition of tyrosine phosphorylation of STAT3, the key effector of the NPM/ALK-induced oncogenesis. We also show that the STAT3 tyrosine phosphorylation is mediated in the malignant T cells by NPM/ALK independently of Jak3 kinase as evidenced by the presence of STAT3 phosphorylation in the NPM/ALK-transfected BaF3 cells that do not express detectable Jak3 and in the NPM/ALK-positive malignant T cells with either Jak3 activity impaired by a pan-Jak or Jak3-selective inhibitor or Jak3 expression abrogated by Jak3 siRNA. The above results represent the 'proof-ofprinciple' experiments with regard to the ALK enzymatic activity as an attractive therapeutic target in T-cell lymphomas and other malignancies that express the kinase in an active form.

Laboratory Investigation (2005) 85, 1544-1554. doi:10.1038/labinvest.3700348; published online 19 September 2005
\end{abstract}

Keywords: ALK; STAT3; Jak3; T-cell lymphoma; kinase inhibitor

T-cell lymphomas comprise a diverse group of biologically and clinically distinct malignancies. Primary cutaneous T-cell lymphoproliferative disorders (CTCL) represent the most frequently occurring type; they display a tendency to progress over time to more malignant forms. T-cell lymphomas that express ALK tyrosine kinase (ALK + TCL) comprise another distinct category that occurs mainly in children and young adults. Ectopic expression of ALK in malignant T-lymphocytes results from various translocations involving ALK gene and several different partners genes, most

Correspondence: Dr MA Wasik, MD, Department of Pathology and Laboratory Medicine, University of Pennsylvania Medical Center, 3400 Spruce Street, 7.106 Founders Pavillion, Philadelphia, PA 19104, USA.

E-mail: wasik@mail.med.upenn.edu

Received 12 May 2005; revised 27 July 2005; accepted 3 August 2005; published online 19 September 2005 frequently the gene that encodes nucleophosmin (NPM). The NPM/ALK chimeric protein contains the oligomerization motif of NPM fused to the cytoplasmic portion of ALK that includes an intact kinase catalytic domain. ${ }^{1-3}$ NPM/ALK is constitutively activated through tyrosine autophosphorylation $^{2,3}$ and displays cell-transforming properties as demonstrated in vitro ${ }^{4-6}$ and in vivo in two different mouse models. ${ }^{7,8}$ Owing to these properties, NPM/ ALK and the other ALK chimeric proteins represent an attractive therapeutic target for small-molecule inhibitors similar to BCR/ABL and other oncogenic tyrosine kinases. ${ }^{9}$

STATs (signal transducers and activators of transcription) are members of the ubiquitously expressed family of transcription factors physiologically activated in response to growth factors and cytokines. ${ }^{10}$ Activation of STATs requires phosphorylation of their key tyrosine residues; the phosphorylated STATs translocate into the 
nucleus and initiate transcription of the growth factor-/cytokine-responsive genes. Persistent activation of one of the STATs, STAT3, seems to play a critical role in the pathogenesis of CTCL, ${ }^{11,12}$ ALK + TCL $^{13,14}$ as well as a large number of other hematopoietic and nonhematopoietic malignancies. ${ }^{15}$ Whereas the signals leading to the continuous STAT3 activation seem to stem in CTCL cells from Jak1/Jak3 tyrosine kinase tandem associated with the cytokine receptor common $\gamma$ chain, ${ }^{11,16}$ in ALK + TCL cells, NPM/ALK itself triggers STAT3 tyrosine phosphorylation ${ }^{13,14}$ with a few reports suggesting an involvement of Jak3 as an intermediary. ${ }^{17,18}$

Here we report that two small-molecule, tyrosine kinase inhibitors designated WHI-P131 (4(4'-hydroxyphenyl)amino-6,7-dimethoxyquinazoline) and WHI-P154 (4-[(3'-bromo-4'-hydroxyphenyl) amino]-6,7-dimethoxyquinazoline) inhibit proliferation and induce apoptotic cell death of the ALK + TCL cells. They abrogate STAT3 tyrosine phosphorylation in the Jak3-independent manner and act by inhibiting directly kinase activity of ALK. Biologic and potential therapeutic implications of these findings are discussed.

\section{Materials and methods}

\section{ALK + TCL, CTCL and BaF3 Cell Lines and Normal Activated Lymphocytes}

SUP-M2 and Karpas 299 cell lines were derived from two different CD30 + anaplastic large T-/nullcell lymphomas and carry the $\mathrm{t}(2 ; 5)$ chromosomal translocation involving ALK and NPM genes as documented by cytogenetic, molecular and/or protein expression analysis. ${ }^{13,14} \mathrm{~PB}-1,-2 \mathrm{~A}$ and $-2 \mathrm{~B}$ T-cell lines were established from a patient with a progressive CTCL. ${ }^{11,12}$ The murine pro-B-cell lymphoid cell line BaF3 was transfected with a vector containing NPM/ALK construct or a control, empty vector. ${ }^{13}$ Normal activated lymphocytes were obtained by mitogen (PHA) stimulation of peripheral blood mononuclear cells (PHA-PBMC) for 6 days followed by $30 \mathrm{~min}$ stimulation with IL-2. The cell populations were cultured at $37^{\circ} \mathrm{C}$ and $5 \% \quad \mathrm{CO}_{2}$ in standard RPMI 1640 medium supplemented with $10 \%$ heat-inactivated fetal bovine serum, $1 \%$ penicillin/streptomycin/fungizone mixture, $2 \mathrm{mM}$ L-glutamine and, in the case of BaF3 cells, $10 \%$ of IL-3 containing WEHI-conditioned medium or recombinant IL-3.

\section{Tyrosine Kinase Inhibitors}

The inhibitors have been reported to display various degree of activity against members of the Jak tyrosine kinase family. As shown in Figure 1, panJak (Jak I; Calbiochem) is a quinolin derivative with the following structure: 2-(1,1-dimethylethyl)-9fluoro-3,6-dihydro-7H-benz[ $h]$-imidaz[4,5- $f]$ isoquinolin-7-one. It inhibits enzymatic function of all four members of the Jak family with the IC50 of $15 \mathrm{nM}$ for Jak1, $1 \mathrm{nM}$ for Jak2, $5 \mathrm{nM}$ for Jak3 and $1 \mathrm{nM}$ for Tyk2 in in vitro kinase activity inhibition assay. ${ }^{19}$ Two other kinase inhibitors, also from Calbiochem, are structurally related quinazoline derivatives (Figure 1): WHI-P131 and WHI-P154. WHI-P134 has been shown, in in vitro kinase inhibition assay, to inhibit selectively Jak3 ( $\left.\mathrm{IC}_{50}: 78 \mu \mathrm{M}\right)$ with no effect on Jak1 and Jak2. ${ }^{20-22}$ WHI-154 inhibits Jak3 ( $\mathrm{IC}_{50}$ : $5,6 \mu \mathrm{M})$ also with no effect on Jak1 and Jak2. ${ }^{20,21,23}$ Another Jak inhibitor that preferentially inhibits Jak3 (the reported $\mathrm{IC}_{50}$ of in vitro kinase inhibitory activity is for Jak3 $1 \mathrm{nM}$, for Jak2 $20 \mathrm{nM}$ and for Jak1 $100 \mathrm{nM}$ ) has been synthesized according to the published structure. ${ }^{24}$ All inhibitors were reconstituted in DMSO to the suitable stock concentration and diluted shortly before the experiments to the final concentrations shown in the figures.

\section{Cell Proliferation and Survival (MTT Enzymatic Conversion) Assay}

Cell lines were seeded in 96-well plates at $1 \times 10^{4}$ cells/well in RPMI medium supplemented with $10 \%$ FBS. After $6 \mathrm{~h}$ culture, the cells were exposed in triplicate cultures to serial dilutions of the

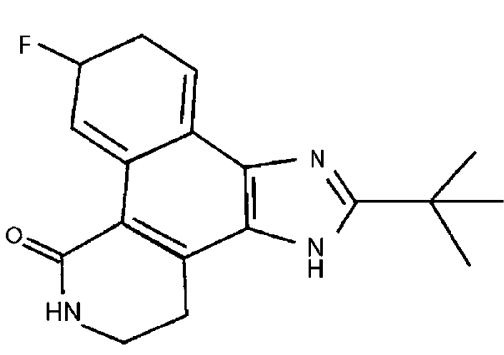

PAN-JAK INHIBITOR

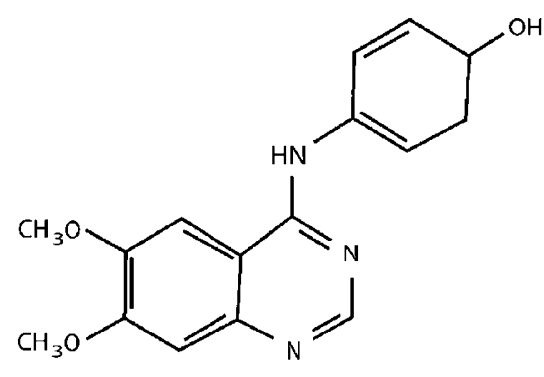

WHI-P131

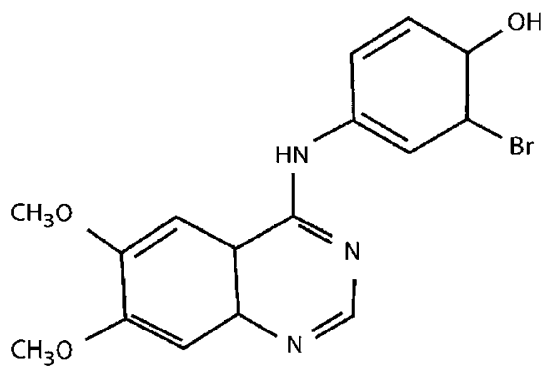

WHI-P154

Figure 1 Chemical structure of the tyrosine kinase inhibitors examined in the study: quinolin derivative, pan-Jak, and quinazoline derivatives, WHI-P131 and WHI-P154. 
tyrosine kinase inhibitors. After $72 \mathrm{~h}$, the relative number of viable cells was determined by the use of MTT reduction colorimetric assay (Promega).

\section{Cell Cycle Analysis}

After the cell culture at $0.5 \times 10^{6}$ cells $/ \mathrm{ml}$ for $24 \mathrm{~h}$ with the tyrosine kinase inhibitors at the concentration depicted in Figure 3, the cells were harvested, washed twice with PBS and permeabilized with $75 \%$ ice-cold ethanol for at least $1 \mathrm{~h}$ at $4^{\circ} \mathrm{C}$. After the permeabilization, the cells were washed twice with PBS and resuspended in Master Mix (950 $\mu$ l PBS, $0.1 \mathrm{mg}$ RNAse; Roche) and $40 \mu \mathrm{g}$ of propidium iodine (PI; Sigma) and incubated for $15 \mathrm{~min}$ at $37^{\circ} \mathrm{C}$. The cells were analyzed by flow cytometry (FACSort BD) using CellQuest Pro and ModFit software.

\section{Cell Apoptosis Assays}

The analysis was performed using two separate experimental approaches. In the first, the intact cells were exposed for $30 \mathrm{~min}$ to the $4 \% \mathrm{PI} / \mathrm{RNAse} / \mathrm{PBS}$ solution and examined by flow cytometry. In the second approach, we used the ApoAlert DNA Fragmentation Assay Kit from BD Bioscience according to the manufacturer's protocol. In brief, after the cell culture at $0.5 \times 10^{6}$ cells $/ \mathrm{ml}$ for $24 \mathrm{~h}$ with the tyrosine kinase inhibitors at the concentration depicted in Figure 3, the cells were collected, washed twice in PBS and fixed with 1\% formaldehyde/PBS. After a wash, the cells were permeabilized with $70 \%$ ice-cold ethanol for at least $2 \mathrm{~h}$, washed and incubated in TdT incubation buffer for $1 \mathrm{~h}$ at $37^{\circ} \mathrm{C}$. The reaction was stopped by adding $20 \mathrm{mM}$ EDTA and the cells were washed twice in $0.1 \%$ Triton X-100/BSA/PBS. Finally, samples were resuspended in $0.5 \mathrm{ml}$ of $\mathrm{PI} / \mathrm{RNAse} / \mathrm{PBS}$, collected and analyzed by Flow Cytometry (FACSort BD) using the CellQuest PRO software.

\section{In Vitro NPM/ALK Kinase Enzymatic Activity Assays}

NPM/ALK tyrosine kinase activity was examined by two separate methods that used a synthetic tyrosinerich substrate and measured the kinase autophosphorylation. The first employed an EIA-based protein tyrosine kinase assay kit (Sigma, St Louis, MO, USA) as described previously. ${ }^{13}$ In brief, cells were lysed with lysis buffer that contained protease and phosphatase inhibitors. Next, immunoprecipitation with the ALK goat polyclonal antibody (Santa Cruz Biotechnology) and $20 \mu \mathrm{l}$ Protein G Agarose (Gibco BRL) was performed. The immunoprecipitates were added to the wells coated with poly-GluTyr substrate. After 30 min incubation, the plates were washed and incubated with a monoclonal anti-phosphotyrosine/peroxidase conjugate. After washing, solution containing peroxidase substrate was applied to the plate. The reaction was stopped by adding $2.5 \mathrm{~N} \mathrm{H}_{2} \mathrm{SO}_{4}$ and color intensity was measured in a microplate EIA reader. The purified human EGF receptor supplied by the kit's manufacturer was used as a positive control.

To determine the NPM/ALK autophosphorylation, aliquots of cells were normalized for cell counts $\left(10 \times 10^{6}\right)$ and the cells were lysed in ice-cold lysis buffer ( $2 \%$ NP-40, $10 \mathrm{mM} / \mathrm{l}$ Tris (pH 7.6), $50 \mathrm{mM} / \mathrm{l}$ $\mathrm{NaCl}, 30 \mathrm{mmol} / \mathrm{l}$ sodium pyrophosphate, $50 \mathrm{mM} / \mathrm{l} \mathrm{NaF}$, $1 \mathrm{mM} / \mathrm{l}$ phenylmethylsulfonylfluoride, $\quad 0.24 \mathrm{U} / \mathrm{ml}$ aprotinin, $10 \mu \mathrm{g} / \mathrm{ml}$ leupeptin, $10 \mu \mathrm{M} / \mathrm{l}$ pepstatin and $1 \mathrm{mM} / \mathrm{l}$ sodium orthovanadate) at $4^{\circ} \mathrm{C}$ for $10 \mathrm{~min}$. Nuclei and cellular debris were removed by centrifugation at $14000 \mathrm{~g}$ for $20 \mathrm{~min}$ at $4^{\circ} \mathrm{C}$. In selected experiments, the lysates were additionally normalized for total protein content with no effect on the results. The kinase assay was performed by incubating NPM/ALK immunoprecipitates in $30 \mu \mathrm{l}$ kinase reaction mixture $(25 \mathrm{mmol} / \mathrm{l}$ HEPES ( $\mathrm{pH} 7.1)$, $10 \mathrm{mmol} / \mathrm{l} \mathrm{MnCl}_{2}, 10 \mu \mathrm{Ci}\left[{ }^{32} \mathrm{P}-\gamma\right]$-ATP, $1 \mu \mathrm{mol} / \mathrm{l}$ unlabeled ATP) for $2-10 \mathrm{~min}$ at $20^{\circ} \mathrm{C}$, as described. ${ }^{25}$

\section{Western Blot}

The cells were washed briefly in PBS, centrifuged and lysed in RIPA buffer $(50 \mathrm{mM}$ Tris-HCl $\mathrm{pH} 7.4$, $1 \% \mathrm{NP}-40,0.25 \%$ sodium deoxycholate, $150 \mathrm{mM}$ $\mathrm{NaCl}, 1 \mathrm{mM}$ EDTA) supplemented with $0.5 \mathrm{mM}$ PMSF, phosphatase inhibitor cocktails I and II from Sigma and protease inhibitor cocktail from Roche, according to the manufacturer's specifications. For normalization of the gel loading, the protein extracts were assayed with Lowry method (Bio-Rad Dc protein assay). Typically, $20-30 \mathrm{mg}$ of the protein per lane was loaded. To examine protein phosphorylation, the membranes were incubated with the antibodies specific for STAT3 phosphorylated on the key tyrosine 705 or ALK phosphorylated on tyrosine positioned as 1604 in the nonchimeric, native protein (both from Cell Signaling). To detect total protein, we used STAT3, STAT5, Jak3 and Jak2 antibodies from Santa Cruz, and Jak1 and Tyk2 antibodies from BD Transduction Laboratories. Next, the membranes were incubated with the appropriate secondary, peroxidase-conjugated antibodies. The blots were developed using the SuperSignal West Dura from Pierce.

\section{Jak3 siRNA Assay}

Mixture of four JAK3-specific siRNA or nonsense siRNA (both purchased from Dharmacon) was introduced into cells at $100 \mathrm{nM}$ by lipofection with the new generation Lipofectamine (DMRIE-C; Invitrogen). The procedure was repeated after $48 \mathrm{~h}$ and the cells were cultured for additional $48 \mathrm{~h}$. The cells were harvested at two time points $(24 \mathrm{~h}, 48 \mathrm{~h})$ after first transfection and at one time point ( $48 \mathrm{~h}$ ) 
after second transfection. The expression of JAK3 was examined by Western blotting.

\section{Results}

WHI-P131 and WHI-P154 Preferentially Inhibit Growth of ALK + TCL Cells

Owing to the reports that suggested Jak3 involvement in ALK signaling, ${ }^{17,18}$ we decided to examine the effect of tyrosine kinase inhibitors that display various degree of anti-Jak3 activity on the ALK + TCL cells in the MTT conversion assay that determines the combination of cell proliferation and survival. CTCL-derived cell lines that display constitutive activation of the cytokine common $\gamma$-chain/Jak1/Jak3 complex ${ }^{11,16}$ served as a control. We have used three different tyrosine kinase inhibitors depicted in Figure 1 and described in Materials and methods: one, a benz-imidaz-isoquinolin derivative, designated pan-Jak, and two other, amino-dimethoxyquinazoline derivatives, designated WHI-P131 and WHI-P154. Pan-Jak inhibits in vitro kinase enzymatic activity of all four known members of the Jak kinase family: Jak1, 2 and 3 and Tyk2 with the $\mathrm{IC}_{50}$ in the $1-15 \mathrm{nM}$ dose range. ${ }^{19}$ WHI-P131 was found to display a weak, but selective, anti-Jak3 activity ( $\mathrm{IC}_{50}$ of $78 \mu \mathrm{M}$; Goodman et $a{ }^{20},{ }^{20}$ Sudbeck et $a l^{21}$ and Tibbles et $a{ }^{22}$ ), whereas WHI-P154 shows the selective anti-Jak3 activity at the much lower doses $\left(\mathrm{IC}_{50}\right.$ of $5.6 \mu \mathrm{M}$; Goodman et $a{ }^{20}{ }^{20}$ Sudbeck et $a l^{21}$ and Ghosh et $a l^{23}$ ). Both these agents are inactive towards Jak1 and Jak2. ${ }^{20-23}$

As shown in Figure 2a, pan-Jak inhibitor displayed a substantial activity against the CTCL cells with $\mathrm{IC}_{50}$ cell growth inhibitory activity of approximately $0.4 \mu \mathrm{M}$. In striking contrast, it had very limited inhibitory effect on the ALK + TCL cells even at the $3 \mu \mathrm{M}$ dose, suggesting that activity of Jak3 and the other members of the Jak family are not critical for proliferation and survival of these cells. In contrast, WHI-P131 and WHI-P154 showed an activity pattern that profoundly differed from the above-mentioned effect of pan-Jak. As shown in Figure $2 \mathrm{~b}$ and $\mathrm{c}$, respectively, they both preferentially inhibited ALK + TCL cells. Whereas WHIP131 inhibited growth of the ALK + TCL cells at $\mathrm{IC}_{50}$ of approximately $10 \mu \mathrm{M}$, it had a much lesser effect on the CTCL cells $\left(\mathrm{IC}_{50}>45 \mu \mathrm{M}\right)$. In turn, WHI-P154 also exerted a greater negative impact on ALK + TCL cells than on CTCL cells, but the difference was less pronounced ( $\mathrm{IC}_{50}$ of 5 and $>14 \mu \mathrm{M}$, respectively).

\section{WHI-P131 and WHI-P154 Inhibit Cell Cycle Progression and Induce Apoptotic Cell Death of ALK + TCL Cells}

As the MTT conversion assay does not distinguish well between changes in cell proliferation and
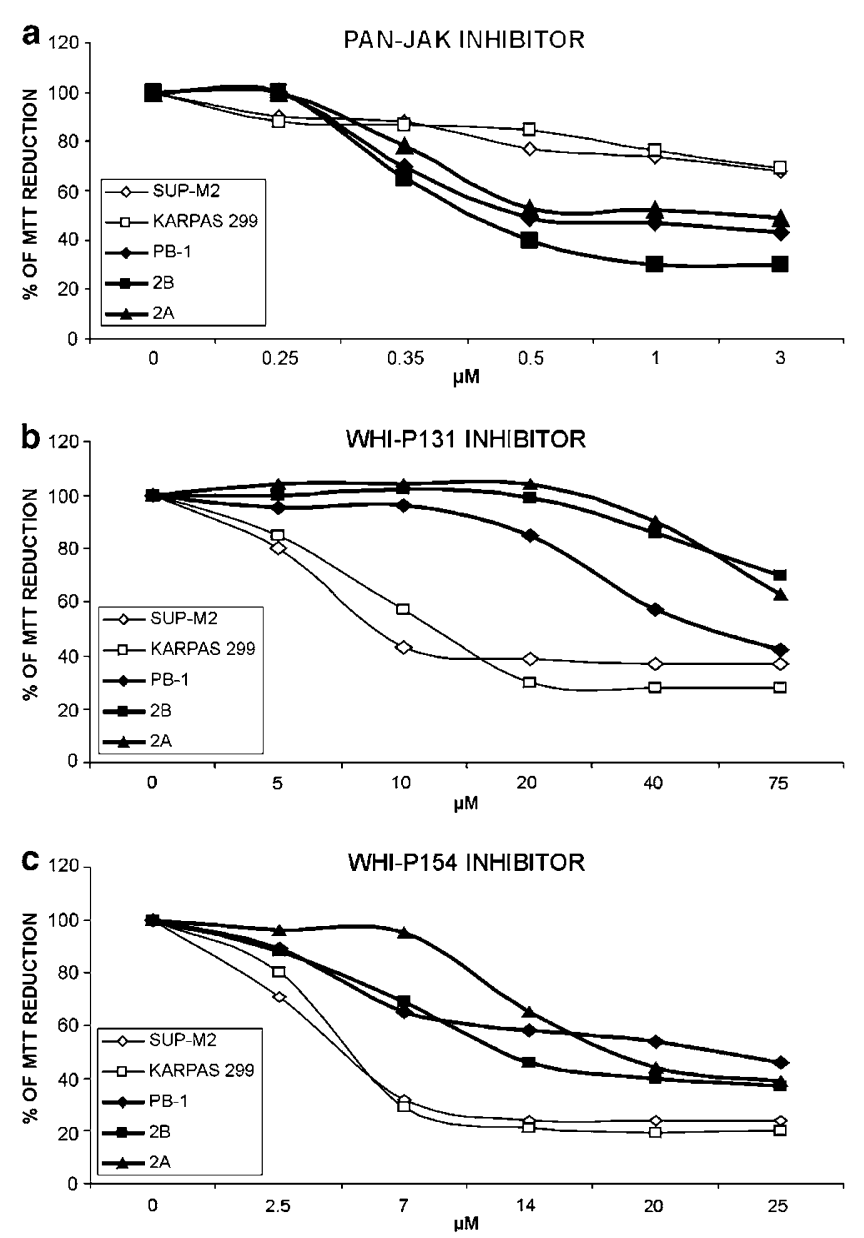

Figure 2 Differential effect of the tyrosine kinase inhibitors: pan-Jak (a) and WHI-P131 (b) and WHI-P154 (c) on growth of malignant T cells. The ALK + TCL (SUP-M2 and Karpas 299) and CTCL (PB-1, -2A, and -2B) cells were cultured in triplicates with medium alone or the inhibitors at the indicated concentrations for $72 \mathrm{~h}$, followed by a $2-3 \mathrm{~h}$ pulse with the MTT substrate. The results are presented as the percentage of the MTT reduction with the untreated cells serving as the reference value (100\%) and are representative of at least four independent experiments.

viability, we examined next the effect of the WHI compounds and pan-Jak inhibitor on cell cycle and necrosis and apoptosis of the ALK + TCL and CTCL cells. As shown in Figure 3a, all agents that were applied at the double $\mathrm{IC}_{50}$ cell growth inhibitory dose suppressed cell cycle progression at the early stage as determined by the accumulation of cells in the G0/G1 phase and the decrease in percent of cells in the S phase. In parallel to the results of the MTT assay, this effect varied among the inhibitors with regard to its degree and preference towards either ALK + TCL or CTCL cells. Accordingly, WHI-P131 was markedly more effective against the former than the latter (30 and $8 \%$ decrease in cells in the S-phase, respectively), whereas WHI-P154 affected ALK + TCL and CTCL cells more comparably (25 vs $15 \%)$. As expected, pan-Jak inhibitor markedly suppressed CTCL cells causing a $40 \%$ decrease in the S-phase cells. It, however, affected also the 

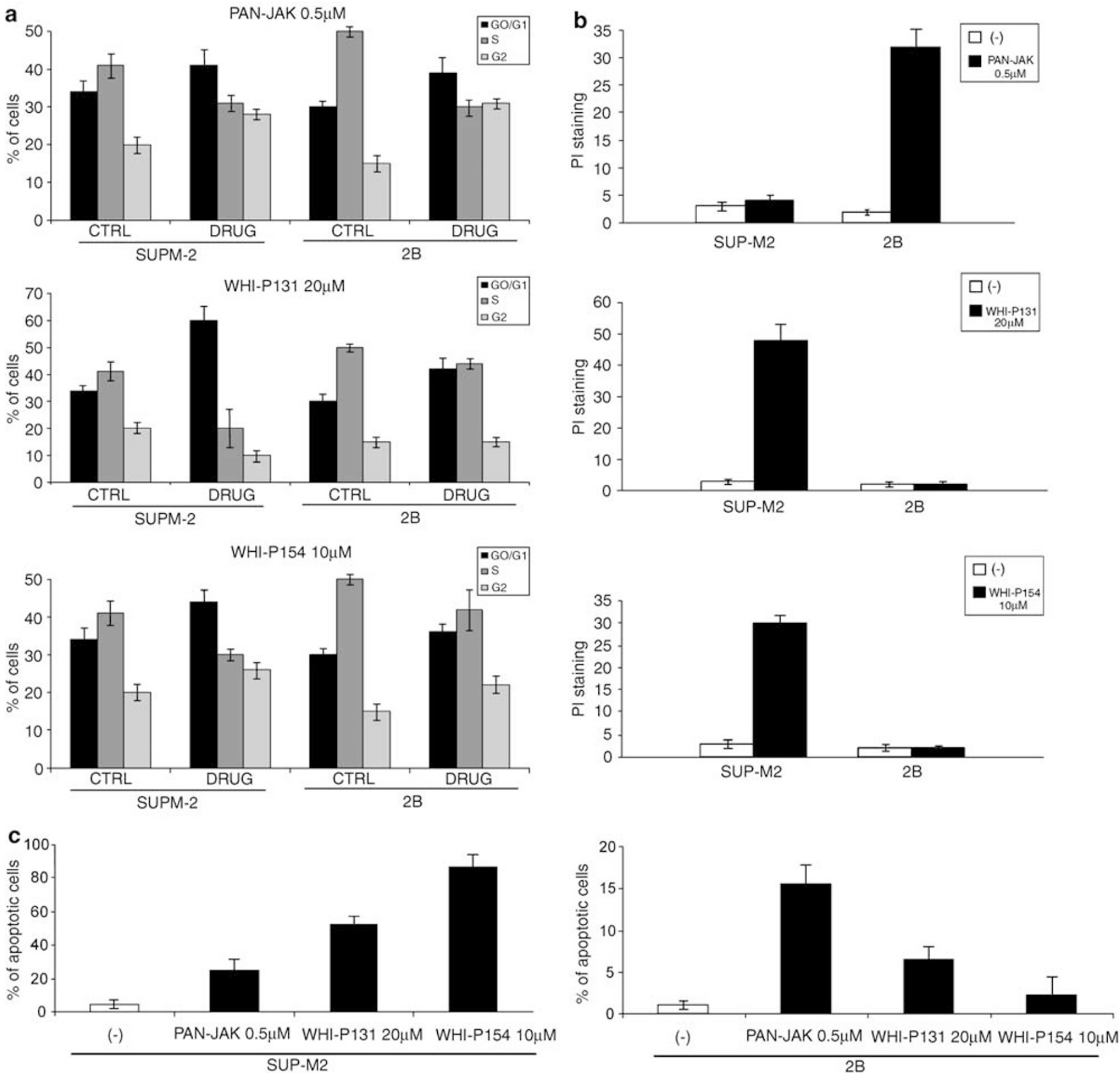

Figure 3 Differential effect of the pan-Jak (a) and WHI-P131 (b) and WHI-P154 (c) inhibitors on the cell cycle progression and survival of the ALK + TCL and CTCL cells. (a) Cell cycle progression as determined by PI incorporation into isolated cell DNA. The results indicate change in the percentage of cells in the G0/G1 (gray rectangles) and S (black rectangles) phase upon cell treatment with the inhibitors. (b) Cell death as determined by PI incorporation into intact cells. The results indicate the percentage of the PI-staining cells upon exposure to medium alone (white rectangles) and the inhibitors at the indicated doses (black rectangles). (c) Cell apoptosis as determined by the DNA fragmentation (Tunel) assay. The results indicate change in the percentage of cells with the DNA breaks upon treatment with the inhibitors at the indicated doses (black rectangles). Cells exposed to the medium alone served as the negative control (white rectangles). All the above experiments are representative of at least three independent experiments.

ALK + TCL cells (25\% decrease), suggesting that the MTT assay that determines combined cell survival and proliferation and has somewhat underestimated the antiproliferative impact of the agent on these cells.

In the same set of flow-cytometry-based experiments, we evaluated changes in PI incorporation by the whole cells as a measure of late apoptosis. As shown in Figure 3b, pan-Jak3 increased cell death rate in CTCL cells by almost $30 \%$. In striking contrast, it displayed no detectable effect on survival of the ALK + TCL cells. Both WHI-P131 and WHI-P154 had the opposite to pan-Jak effect of inducing death in $>45$ and $30 \%$ of the ALK + TCL cells, respectively, and leaving the CTCL apparently unaffected. To evaluate apoptotic cell death more accurately, we have performed a DNA fragmentation (Tunel) assay. As shown in Figure 3c, the outcome 
was overall similar to the PI staining results with CTCL cells being affected preferentially by the pan-Jak3 inhibitor and the ALK + TCL cells by the WHI compounds. Noteworthy, WHI-P154-induced apoptotic rate of the ALK + TCL was as high as $80 \%$ with minimal, if any, effect on the CTCL cells.

WHI-P131 and WHI-P154, but not Pan-Jak Inhibitor, Abrogate STAT3 Phosphorylation in ALK + TCL Cells

As phosphorylation of STAT3 is the major result of NPM/ALK-mediated malignant T-cell transformation $^{9,13,14,26}$ with some suggestions that it might occur through activation of Jak3 kinase, ${ }^{17,18}$ we examined next the effect of pan-Jak and WHI inhibitors on STAT3 activation as determined by its phosphorylation at the key tyrosine 705. As shown in Figure 4a, pan-Jak had no effect on the STAT3 tyrosine phosphorylation in the ALK + TCL cells even at the highest dose of $3 \mu \mathrm{M}$ examined, whereas it markedly inhibited the STAT3 phosphorylation in CTCL cells at the 0.1-0.5 $\mu \mathrm{M}$ dose range. In striking contrast, WHI-P131 and WHI-P154 inhibited in the dose-dependent manner STAT3 phosphorylation in the ALK + TCL but not CTCL cells (Figure $4 \mathrm{~b}$ and c, respectively).

\section{Jak3-Independent Phosphorylation of STAT3 in NPM/ALK-Transfected BaF3 Cells}

To provide direct evidence that the noted drug effect on STAT3 tyrosine phosphorylation in ALK + TCL cells (Figure 4) is Jak3 independent, we examined STAT3 activation in BaF3 cells transfected with NPM/ALK. BaF3 cells transfected with an empty vector served as a negative control. As shown in Figure 5a (left panel), expression of NPM/ALK results in STAT3 phosphorylation in agreement with our previous findings. ${ }^{13}$ Noteworthy, the transfected $\mathrm{BaF} 3$ do not express Jak3, although they express the other, more ubiquitously expressed members of the Jak family: Jak1, Jak2 and Tyk2 (Figure 5a, right panel). The above finding indicates that NPM/ALK induces STAT3 phosphorylation in the BaF3 cells independently of Jak3.

\section{WHI-P131 and WHI-P154, but not Pan-Jak Inhibitor, Suppress STAT3 and NPM/ALK Phosphorylation in NPM/ALK-Transfected BaF3 Cells}

We evaluated next the effect of the three tyrosine kinase inhibitors in the NPM/ALK-transfected BaF3 a

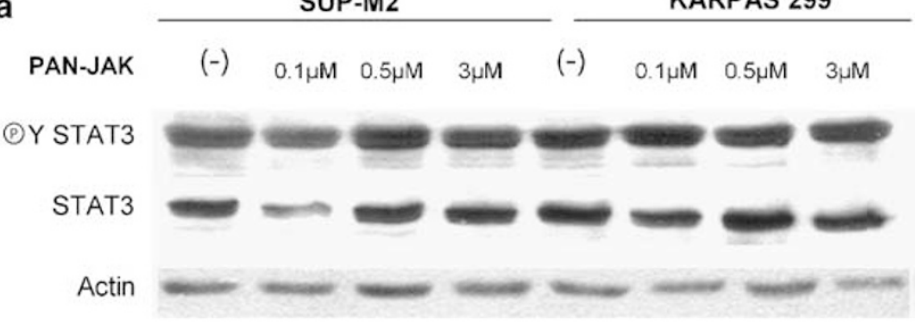

PB-1

$(-) \quad 0.1 \mu \mathrm{M} \quad 0.5 \mu \mathrm{M} \quad 3 \mu \mathrm{M}$

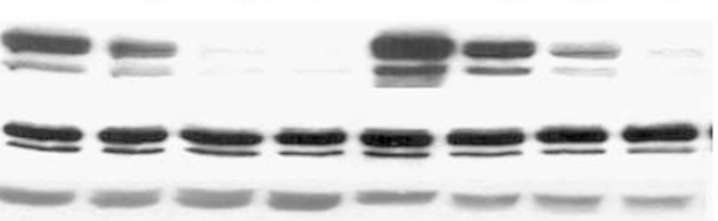

b

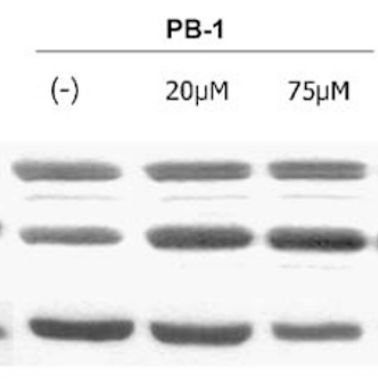

$2 \mathrm{~B}$
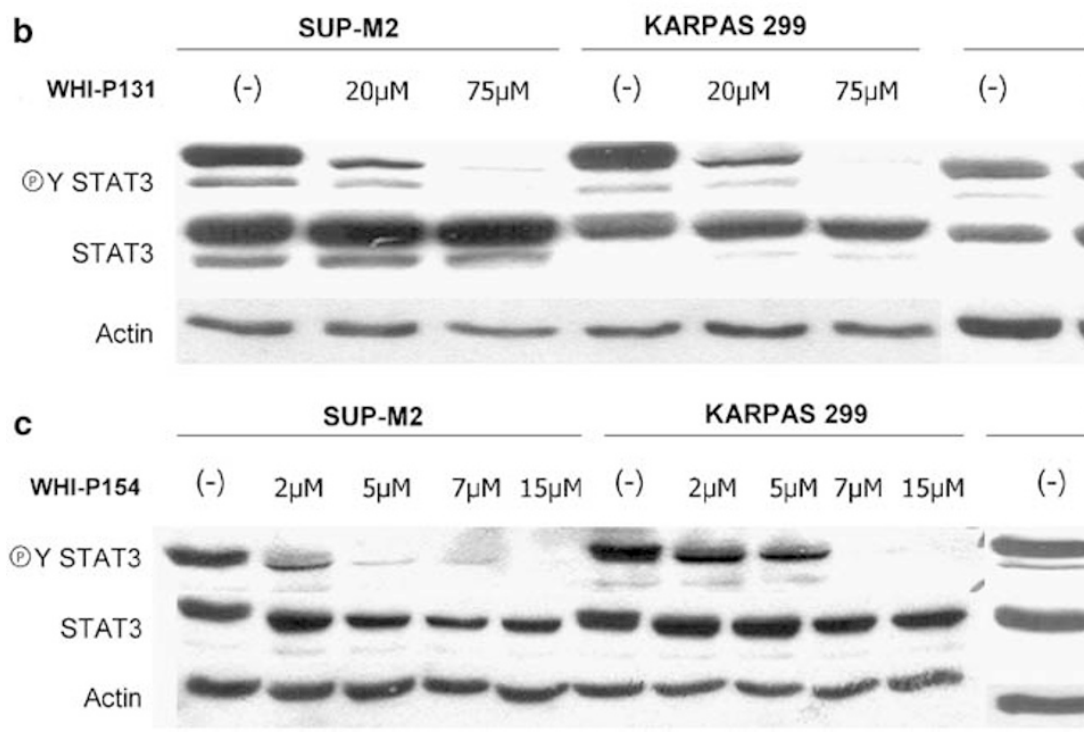

PB-1

2B

Figure 4 Differential effect of the pan-Jak (a) and WHI-P131 (b) and WHI-P154 (c) inhibitors on STAT3 tyrosine phosphorylation in the ALK + TCL and CTCL cells. The cells were treated with the inhibitors at the indicated doses for $1 \mathrm{~h}$, lysed and evaluated by Western blotting for expression of phospho(Tyr705)-STAT3; total STAT3 and actin served as controls. The results are representative of three independent experiments. 
a

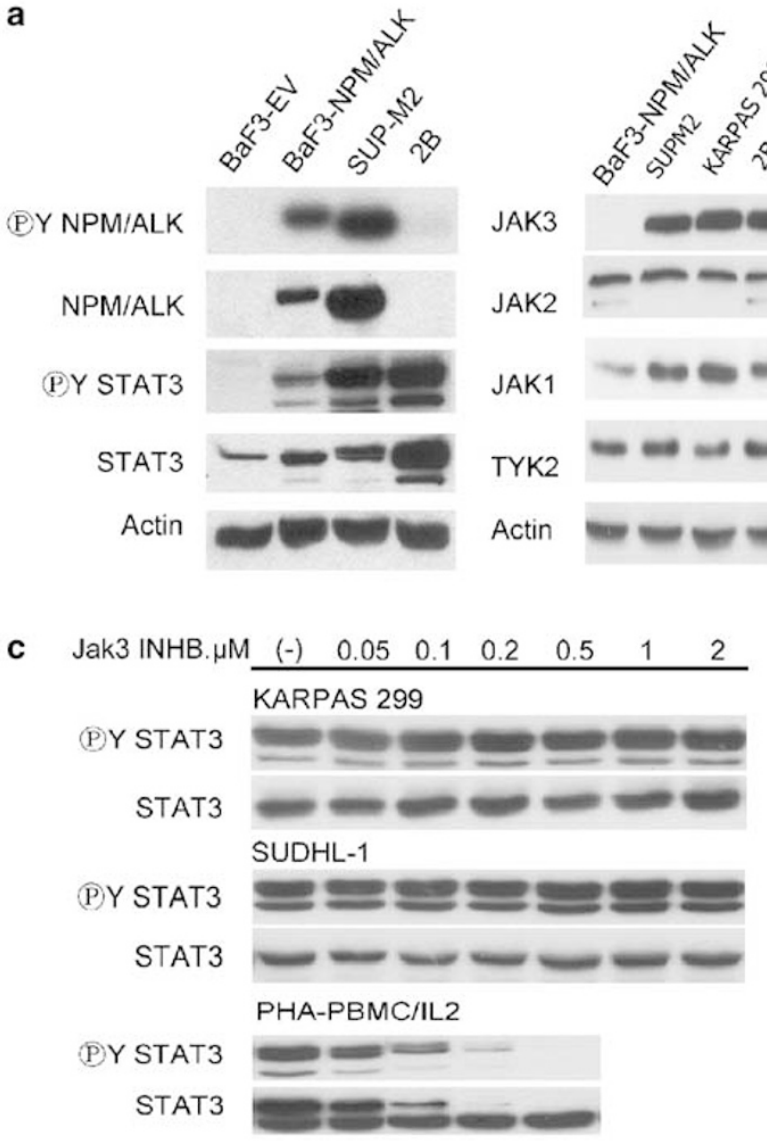

b

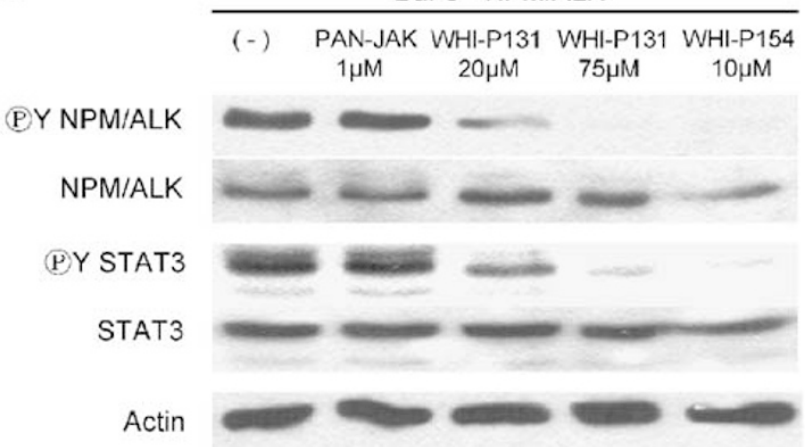

d

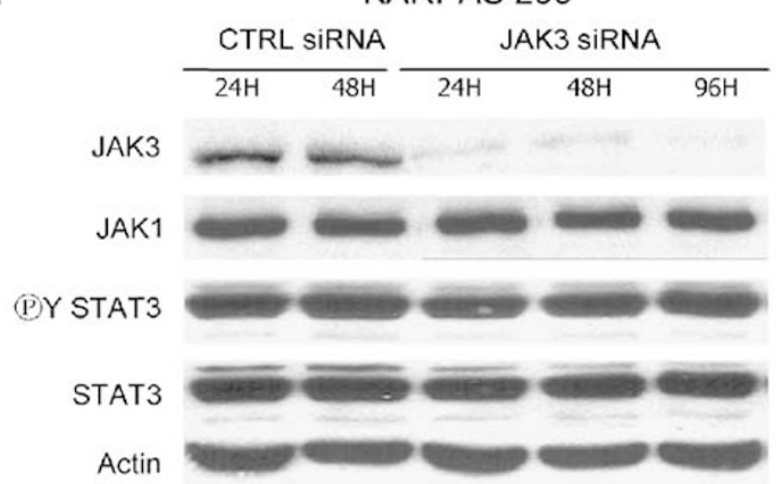

Figure 5 Lack of involvement of Jak3 in STAT3 tyrosine phosphorylation in the NPM/ALK-expressing cells. (a) Expression of phosphoSTAT3 and Jak family proteins in the NPM/ALK-transfected cells. Immature B-cell lymphoid BaF3 cells were transfected with the NPM/ ALK construct ${ }^{14}$ or empty vector and evaluated by Western blot for the expression of NPM/ALK, STAT3 (in the tyrosine-phosphorylated and total form) and Jak3 and the other three members of the Jak kinase family. ALK + TCL cells (SUP-M2 and Karpas 299) and CTCL cells (2B) served as controls. (b) Differential effect of the pan-Jak and WHI-P131 and WHI-P154 inhibitors on NMP/ALK and STAT3 tyrosine phosphorylation in the NPM/ALK-transfected BaF3 cells. The cells were treated with the inhibitors at the indicated doses for $1 \mathrm{~h}$, lysed and evaluated by Western blotting for the expression of phospho-NPM/ALK and phospho-STAT3; total NPM/ALK, STAT3 and actin served as controls. (c) Effect of Jak3 inhibition on STAT3 phosphorylation. The ALK + TCL cells Karpas 299 and SUDHL-1 and control, PHA preactivated, IL-2 stimulated and normal PBMC (PHA-PBMC/IL-2) were cultured for $1 \mathrm{~h}$ with selective Jak3 inhibitor at the indicated doses. Expression of phospho(Tyr705)-STAT3 and total STAT3 was determined by Western blotting. (d) Effect of downregulation of Jak3 expression on STAT3 phosphorylation. The Karpas 299 cells were lipotransfected with $100 \mathrm{nM}$ of Jak3 siRNA pool or control (CTRL) nonsense siRNA at times 0 and $48 \mathrm{~h}$ and lysed at 24, 48 and $96 \mathrm{~h}$. Expression of Jak3, phospho-STAT3, Jak1, total STAT3 and actin (the last three serving as controls) was determined by Western blotting. All the above experiments are representative of at least three independent experiments.

cells on phosphorylation of STAT3 and NPM/ALK. As shown in Figure 5b, both WHI-P131 and WHIP154 inhibited STAT3 phosphorylation with the latter being somewhat more effective similar to their effect in the ALK + TCL cells (Figure 4). Remarkably, the agents also inhibited phosphorylation of NPM/ALK at the same doses, indicating that they are active against the NPM/ALK kinase itself. In contrast, pan-Jak inhibitor had no effect on either STAT3 or NPM/ALK tyrosine phosphorylation leading, in combination with the ALK + TCL result, to the conclusion that NPM/ALK might phosphorylate STAT3 directly without any detectable contribution of not only Jak3 but also other members of the Jak kinase family.

\section{Jak3 Enzymatic Inactivation or Depletion does not Affect STAT3 Phosphorylation in ALK + TCL Cells}

To provide the direct evidence that Jak3 is not required for STAT3 tyrosine phosphorylation also in the ALK + TCL cells, we employed two different experimental approaches. First, we incapacitated Jak3 functionally using a recently described ${ }^{24}$ smallmolecule inhibitor that is highly selective for the kinase (the reported $\mathrm{IC}_{50}$ of in vitro kinase inhibitory activity is for Jak3 $1 \mathrm{nM}$, for Jak2 $20 \mathrm{nM}$ and for Jak1 $100 \mathrm{nM}$, and $\mathrm{IC}_{50}$; with the in vivo inhibitory activity of approximately 50-fold higher). As shown in Figure 5c, the Jak3 inhibitor had no effect on in vivo STAT3 phosphorylation at the functionally 
key tyrosine 705 in the ALK + T-cell lines Karpas 299 and SUDHL-1 even at $2 \mu \mathrm{M}$. In contrast, it markedly inhibited such STAT3 phosphorylation in the control, mitogen (PHA) and IL-2-stimulated PBMCs at 100-200 nM with the complete inhibition achieved at $500 \mathrm{nM}$. In the second approach, we utilized Jak3-specific siRNA with nonsense siRNA serving as a negative control. The Jak3 siRNA essentially abrogated expression of the Jak3 protein without affecting expression of the closely related protein Jak1 and the other two control proteins total STAT3 and actin as depicted in Figure 5d. Importantly, the Jak3 depletion had no effect on the STAT3 phosphorylation. As expected, the nonsense siRNA did not affect expression of Jak3, phospho-STAT3 and the other proteins. These results support the conclusion that STAT3 is activated by NPM/ALK without involvement of Jak3.

\section{WHI-P131 and WHI-P154, but not Pan-Jak, Directly Inhibit Kinase Activity of NPM/ALK}

To provide direct evidence that WHI-P131 and WHIP154 inhibits NPM/ALK, we determined the effect of the compounds on enzymatic activity of the NPM/ALK kinase in these cells by performing three different types of experiments. We examined first the drug effect on NPM/ALK tyrosine phosphorylation that is known to result from the kinase autophosphorylation. ${ }^{2,3}$ As shown in Figure 6, panJak had no effect on the phosphorylation at any of the doses tested $(0.1-3 \mu \mathrm{M})$. In contrast, both WHIP131 and WHI-P154 inhibited the NPM/ALK phosphorylation in the dose-dependent manner. Noteworthy, the doses required for marked to complete inhibition matched the ones needed to inhibit STAT3 phosphorylation (Figure 4) and cell survival and proliferation (Figures 2 and 3), supporting the notion that inhibition of NPM/ALK is the main, if not the only, mode of action of these compounds in ALK + TCL cells. In the next set of experiments, we determined in vitro tyrosine kinase activity of NPM/ ALK using a tyrosine-rich oligopeptide substrate ${ }^{13}$ and cell lysate immunoprecipitates obtained with an ALK antibody. As shown in Figure 7a, NPM/ALK kinase activity was markedly inhibited by both WHI-P131 and WHI-P154 with IC $_{50}$ of 10 and $5 \mu \mathrm{M}$, respectively, but remained unaffected by $1 \mu \mathrm{M}$ (>double $\mathrm{IC}_{50}$ dose) of pan-Jak inhibitor. Noteworthy, the $\mathrm{IC}_{50}$ values for both WHI compounds matched the ones required to inhibit cell growth (Figure 1).

Finally, to provide additional evidence that the WHI-P131 and WHI-P154 compounds inhibited NPM/ALK directly, we performed an in vitro autophosphorylation of the purified NPM/ALK. As shown in Figure $7 \mathrm{~b}$, the results closely resembled the ones obtained with the synthetic substrate (Figure 7a). Accordingly, pan-Jak had no effect on the phosphorylation, whereas both WHI compounds

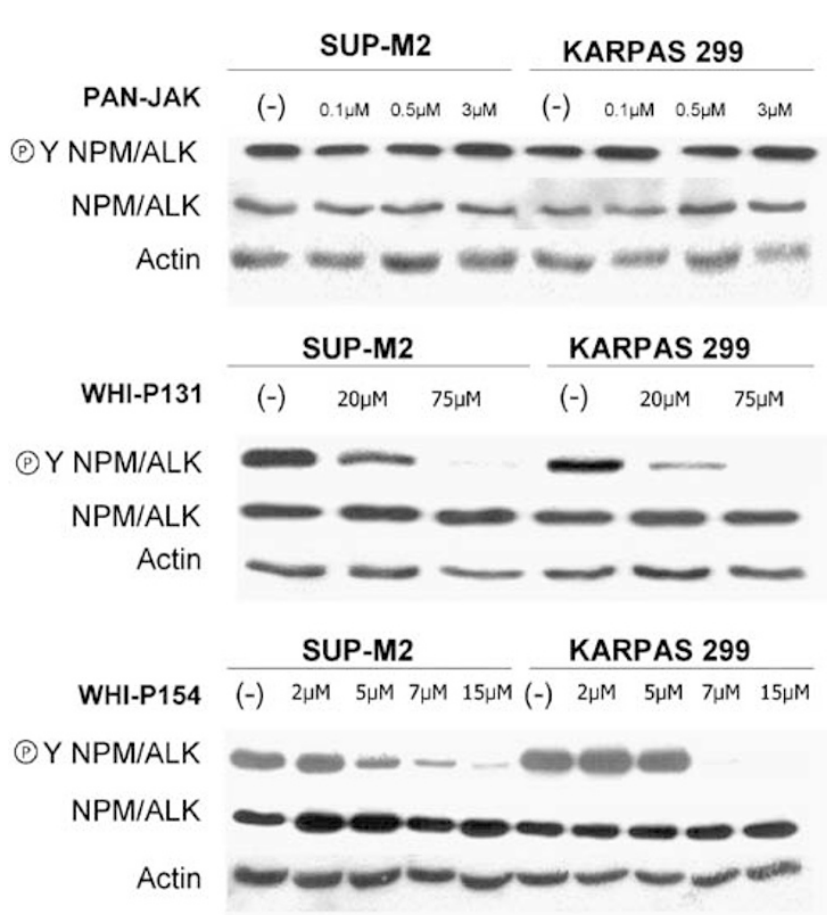

Figure 6 WHI-P131 and WHI-P154 inhibitor-mediated suppression of the NPM/ALK tyrosine phosphorylation in the ALK + TCL cells. The cells were treated with pan-Jak (upper panel), WHI-P131 (middle panel) and WHI-P154 (lower panel) inhibitors at the indicated doses. Cell lysates were evaluated for expression of phospho-NPM/ALK; total NPM/ALK and actin served as controls. The results are representative of at least three independent experiments.

inhibited it markedly. Since we detected in this assay only a single protein that had a molecular weight of $80 \mathrm{kDa}$ characteristic of NPM/ALK, this finding confirms the NPM/ALK identity as the inhibited kinase and, in addition, argues against the presence in the ALK immunoprecipitates of any other activated kinases in the relatively significant amount.

\section{Discussion}

The results in patients with chronic myelogeneous leukemia and related leukemias that carry the chimeric BCR/ABL tyrosine kinase were the first to demonstrate that the constitutively activated, chimeric kinases represent an attractive therapeutic target. ${ }^{27}$ Similar to BCR/ABL, NPM/ALK is oncogenic in vitro ${ }^{4-6}$ and in vivo in transgenic mouse models. ${ }^{7,8}$ Therefore, inhibition of enzymatic activity of NPM/ALK and the other ALK fusion proteins $^{9}$ should lead to a potent antitumor effect and be potentially beneficial for patients with not only ALK + TCL but also other malignancies such as inflammatory myofibroblastic tumors ${ }^{28,29}$ in which the chimeric ALK appears to play a key role in their pathogenesis. ${ }^{9}$ Expression of ALK in its native, apparently structurally intact form has been 
a

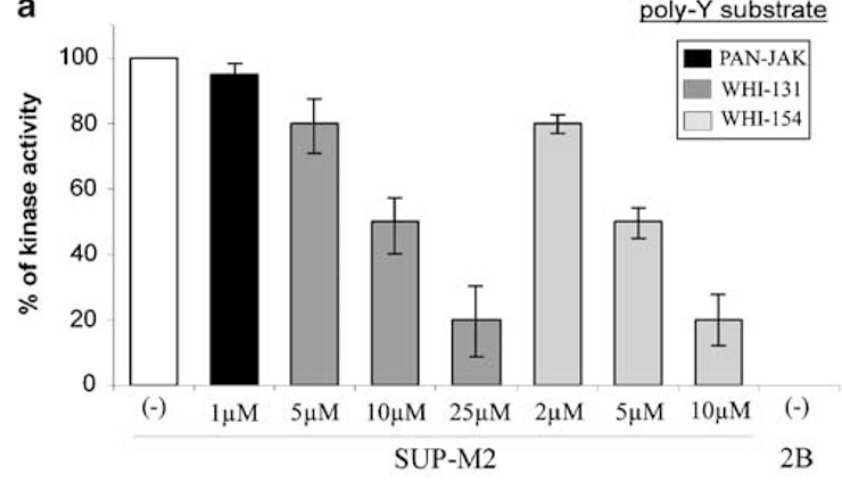

b

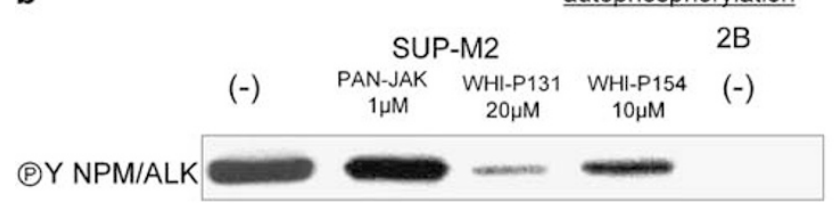

Figure 7 WHI-P131 and WHI-P154 inhibitor-mediated suppression of enzymatic activity of the NPM/ALK tyrosine kinase. (a) In vitro kinase activity of NPM/ALK as detected by phosphorylation of the tyrosine-rich substrate. Protein lysates from the ALK + TCL (SUP-M2) cells exposed for $1 \mathrm{~h}$ to either medium alone (white rectangle) or the pan-Jak and WHI-P131 and WHI-P154 inhibitors (black rectangles) at the depicted doses were immunoprecipitated with the anti-ALK antibody followed by detection of phosphorylation of the synthetic poly-Glu-Tyr peptide in EIA. CTCL cell line 2B served as a negative control. The presented data are representative of four independent experiments. (b) In vitro NPM/ALK autophosphorylation. The ALK + TCL (SUP-M2) cell lysates were immunoprecipitated with an NPM/ALK antibody and incubated for $5 \mathrm{~min}$ at $37^{\circ} \mathrm{C}$ with the kinase inhibitors at the indicated doses or the control buffer in the presence of radioactive $\left[{ }^{32} \mathrm{P}-\gamma\right]$-ATP. 2B cells were used as negative control. The results are representative of two independent experiments.

identified in a number of malignancies, mostly of the neural origin; however, contribution of ALK to their neoplastic phenotype remains to be determined. ${ }^{9}$ We demonstrate in this report that, in contrast to the quinolin-type pan-Jak inhibitor, two quinazoline derivatives, WHI-131 and WHI-154, exert a profound inhibitory effect on the ALK+ TCL-derived cells. These compounds inhibit cell cycle progression in the NPM/ALK-expressing malignant $\mathrm{T}$ cells. Notably from the potential therapeutic perspective, the compounds also induce in such cells extensive apoptosis. On the biochemical level, they essentially abrogate tyrosine phosphorylation of STAT3, the key effector of the NPM/ ALK-mediated oncogenicity. ${ }^{13,14,26}$ Importantly, the compounds directly inhibit NPM/ALK enzymatic activity as demonstrated by in vivo inhibition of NPM/ALK phosphorylation and by in vitro kinase assays either using a tyrosine-rich oligopeptide as the substrate or measuring the degree of NPM/ALK autophosphorylation. The ability of WHI-P131 and WHI-P154 to inhibit STAT3 705 tyrosine phosphorylation in NPM/ALK + TCL cell lines has been noted also by other investigators. ${ }^{17}$ Although their inter- pretation was that this effect was due, at least in part, to inhibition of Jak3, the prevailing data indicate that the STAT3 phosphorylation and ensuing activation are Jak3 independent as discussed in detail below. Our finding that WHI compounds inhibit enzymatic activity of ALK (Figures 5b, 6 and 7) at the concentrations that impair also signal transduction (Figure 4) and key functions (Figures 2 and 3) of the NPM/ALK + TCL cells clearly indicate that the effect of the compounds on the STAT3 phosphorylation should be ascribed mostly, if not exclusively, to the direct inhibition of ALK.

Although WHI-P131 and WHI-P154 provided the 'proof-of-principle' evidence of the validity of targeting NPM/ALK directly, they required the relatively high doses to inhibit growth of the ALK + TCL cells ( IC $_{50}$ of 10 and $5 \mu \mathrm{M}$ for WHI-131 and WHI154, respectively; Figure 2). This may limit their applicability in vivo. However, at the very least the compounds should be very useful as pharmacologic tools to study ALK-mediated oncogenesis. Furthermore, they may provide a lead with regard to structure of the pharmacologically higher-grade agents. Accordingly, addition of a single bromide moiety to the WHI-P154 compound (Figure 1) resulted in the two-fold decrease in the $\mathrm{IC}_{50}$ as compared to the WHI-P131 (Figure 2). In principle, the reported ${ }^{19-23}$ and the apparently noted (Figure 2) crossreactivity of the compounds, in particular of WHI-154, with Jak3 might also be of concern with regard to the potential clinical use of drugs from this class. However, at the current stage, crossreactivity seem to be the feature of essentially all pharmacologic grade kinase inhibitors with the notable exception of rapamycin and its derivatives that target mTOR serine/threonine kinase $^{30}$ by the different mechanism than the tyrosine kinase inhibitors that interfere with binding of ATP within the kinase catalytic domain. Accordingly, imatinib mesylate (STI-571, gleevec) that very effectively targets BCR/ABL kinase in vivo inhibits at similar concentration several other kinases including wildtype ABL, c-kit, PDGFR $\beta$, PDGFR $\alpha^{27}$ and, as shown recently, Syk. ${ }^{31}$ Even the new generation BCR/ABL inhibitor that seems to bind the activated form of the kinase with the much higher affinity, and is aimed at circumventing resistance to imatinib, displays crossreactivity with at least one other tyrosine kinase, src. ${ }^{32}$

The major controversy in the field seems to be the question if NPM/ALK activates STAT3 and other signaling proteins directly or via activation of other kinases, in particular, Jak3. ${ }^{17,26}$ Recent study ${ }^{33}$ that utilized tandem mass spectrometry indicates that NPM/ALK might interact with as many as 46 different proteins that include Jak3. Our current study provides several lines of evidence that NPM/ ALK phosphorylates STAT3 in the Jak3-independent manner. First, pan-Jak inhibitor, in striking contrast to the CTCL cells, has no effect on proliferation, survival and STAT3 phosphorylation 
in the ALK + TCL cells (Figures 2-4). Second, the WHI compounds, in particular WHI-P131, significantly inhibit STAT3 phosphorylation in the ALK + TCL cells at the doses that are approximately fourfold lower than the one required to inhibit Jak3 kinase activity in vitro (Figure 4; Goodman et al, ${ }^{20}$ Sudbeck et $a l^{21}$ and Tibbles et $a l^{22}$ ) which, in turn, is typically 10 -fold lower than the activity in intact cells. Third, transfection with NPM/ALK of the murine precursor B-cell line BaF3 that does not express detectable Jak3 results in STAT3 phosphorylation (Figure 5a). Furthermore, inhibition of Jak3 activity in the ALK + TCL cells by a specific inhibitor $^{24}$ had no effect on STAT3 phosphorylation (Figure 5c). Finally, inhibition in such cells of Jak3 expression by the Jak3-specific siRNA has no impact on the STAT3 phosphorylation despite being very effective in abrogating the Jak3 expression (Figure 5d). Although we cannot totally exclude that another tyrosine kinase different from Jak3 might act as an intermediary in the STAT3 phosphorylation considering the ability of STAT3 to become activated by a rather diverse set of the tyrosine kinases that belong to the growth factor receptor, src and Jak kinase families, ${ }^{15}$ we find it unlikely. First, the lack of any effect of the pan-Jak inhibitor on the ALK + TCL cells (Figures 1-4) rules out the involvement of any member of this family that, beside Jak3, includes Jak1, Jak2 and Tyk2. ${ }^{34}$ Furthermore, two SRC family inhibitors, SU6656 and PP2, used at the doses of up to 1 and $2 \mu \mathrm{M}$, respectively, also had no effect on the Stat3 phosphorylation and cell growth (MTT conversion assay) of the ALK + TCL Karpas 299 cells (data not shown). This observation corroborates the previous finding that mouse embryonal fibrobast cell line devoid of three members of src family known to activate STAT3 were able to display STAT3 phosphorylation upon transfection with NPM/ALK. ${ }^{14}$ Finally, the fact that NPM/ALK is a potent tyrosine kinase that associates with STAT3 as shown by coprecipitation and Western blotting ${ }^{13}$ and co-precipitation and mass spectrometry, ${ }^{33}$ and is by far the most dominant phosphorylated protein in the ALK antibody precipitates (Figure 7b), suggest that NPM/ ALK phosphorylates STAT3 directly. This conclusion is further supported by the lack of any effect on the NPM/ALK-triggered STAT3 phosphorylation of deletional mutation of NPM/ALK that precluded its binding to Jak3. ${ }^{14}$

In summary, we show that the direct inhibition of NPM/ALK by small-molecule, quinazoline-type compounds has a profound suppressive effect on signal transduction, proliferation and survival of the ALK + TCL cells. We also provide evidence that activation of STAT3, the key effector of the NPM/ ALK-induced oncogenesis, is Jak3 independent and likely mediated directly by NPM/ALK itself. The above findings might impact on the development of novel, targeted therapies for ALK + TCL and the other ALK-driven malignancies.

\section{Acknowledgements}

This work was supported in part by grants from the NCI R01-CA89194 and R01-CA96856 (to MAW) and The Danish Cancer Society (to NO).

\section{References}

1 Morris SW, Kirstein MN, Valentine MB, et al. Fusion of a kinase gene, ALK, to a nucleolar protein gene, NPM, in non-Hodgkin's lymphoma. Science 1994;263: 1281-1284.

2 Shiota M, Fujimoto J, Semba T, et al. Hyperphosphorylation of a novel $80 \mathrm{kDa}$ protein-tyrosine kinase similar to Ltk in a human Ki-1 lymphoma cell line, AMS3. Oncogene 1994;9:1567-1574.

3 Morris SW, Naeve C, Mathew P, et al. ALK, the chromosome 2 gene locus altered by the $t(2 ; 5)$ in non-Hodgkin's lymphoma, encodes a novel neural receptor tyrosine kinase (LTK). Oncogene 1997;14: 2175-2188.

4 Bischof D, Pulford K, Mason DY, et al. Role of the nucleophosmin (NPM) portion of the non-Hodgkin's lymphoma-associated NPM-anaplastic lymphoma kinase fusion protein in oncogenesis. Mol Cell Biol 1997;17:2312-2325.

5 Fujimoto J, Shiota M, Iwahara T, et al. Characterization of the transforming activity of p80, a hyperphosphorylated protein in a Ki-1 lymphoma cell line with chromosomal translocation t(2;5). Proc Natl Acad Sci USA 1996;93:4181-4186.

6 Wellmann A, Doseeva V, Butscher W, et al. The activated anaplastic lymphoma kinase increases cellular proliferation and oncogene up-regulation in rat 1a fibroblasts. FASEB J 1997;11:965-972.

7 Chiarle R, Gong JZ, Guasparri I, et al. NPM-ALK transgenic mice spontaneously develop T-cell lymphomas and plasma cell tumors. Blood 2003;101:19191927.

8 Kuefer MU, Look AT, Pulford K, et al. Retrovirusmediated gene transfer of NPM-ALK causes lymphoid malignancy in mice. Blood 1997;90:2901-2910.

9 Wasik MA. Expression of anaplastic lymphoma kinase (ALK) in non-Hodgkin's lymphomas and other malignancies: biological, diagnostic and clinical implications. Am J Clin Pathol 2002;118:S81-S92.

10 Rawlings JS, Rosler KM, Harrison DA. The Jak/STAT signaling pathway. J Cell Sci 2004;117:1281-1283.

11 Zhang Q, Nowak I, Vonderheid EC, et al. Activation of Jak/STAT proteins involved in signal transduction pathway mediated by receptor for interleukin 2 in malignant $\mathrm{T}$ lymphocytes derived from cutaneous anaplastic large T-cell lymphoma and Sezary syndrome. Proc Natl Acad Sci USA 1996;93:9148-9153.

12 Nielsen M, Kaltoft K, Nordahl M, et al. Constitutive activation of a slowly migrating isoform of Stat3 in mycosis fungoides: tyrphostin AG490 inhibits Stat3 activation and growth of mycosis fungoides tumor cell lines. Proc Natl Acad Sci USA 1997;94:67646769.

13 Zhang Q, Raghunath PN, Xue L, et al. Multilevel dysregulation of STAT3 activation in anaplastic lymphoma kinase-positive T/null-cell lymphoma. J Immunol 2002;168:466-474.

14 Zamo A, Chiarle R, Piva R, et al. Anaplastic lymphoma kinase (ALK) activates Stat3 and protects hemato- 
poietic cells from cell death. Oncogene 2002;21: 1038-1047.

$15 \mathrm{Yu} \mathrm{H}$, Jove R. The STATs of cancer-new molecular targets come of age. Nat Rev Cancer 2004;4:97-105.

16 Zhang Q, Raghunath PN, Vonderheid E, et al. Lack of phosphotyrosine phosphatase SHP-1 expression in malignant $\mathrm{T}$ cells results from methylation of the SHP-1 promoter. Am J Pathol 2000;157:1137-1146.

17 Amin HM, Medeiros LJ, Ma Y, et al. Inhibition of JAK3 induces apoptosis and decreases anaplastic lymphoma kinase activity in anaplastic large cell lymphoma. Oncogene 2003;22:5399-5407.

18 Cho-Vega JH, Rassidakis GZ, Amin HM, et al. Suppressor of cytokine signaling 3 expression in anaplastic large cell lymphoma. Leukemia 2004;18:18721878.

19 Thompson JE, Cubbon RM, Cummings RT, et al. Photochemical preparation of a pyridone containing tetracycle: a Jak protein kinase inhibitor. Bioorg Med Chem Lett 2002;12:1219-1223.

20 Goodman PA, Niehoff LB, Uckun FM. Role of tyrosine kinases ininduction of the c-jun proto-oncogene in irradiated B-lineage lymphoid cells. J Biol Chem 1998; 273:17742-17748.

21 Sudbeck EA, Liu XP, Narla RK, et al. Structure-based design of specific inhibitors of Janus kinase 3 as apoptosis-inducing antileukemic agents. Clin Cancer Res 1999;5:1569-1582.

22 Tibbles HE, Vassilev A, Wendorf H, et al. Role of a JAK3-dependent biochemical signaling pathway in platelet activation and aggregation. J Biol Chem 2001; 276:17815-17822.

23 Ghosh S, Jennissen JD, Liu XP, et al. 4-[3-Bromo-4hydroxyphenyl)amino]-6,7-dimethoxyquinazolin-1-ium chloride methanol solvate and 4-[(3-hydroxyphenyl) amino]-6,7-dimethoxy-1-quinazolinium chloride. Acta Crystallogr C 2001;57:76-78.
24 Changelian PS, Flanagan ME, Ball DJ, et al. Prevention of organ allograft rejection by a specific Janus kinase 3 inhibitor. Science 2003;30:875-878.

25 Ptasznik A, Traynor-Kaplan A, Bokoch GM. G proteincoupled chemoattractant receptors regulate Lyn tyrosine kinase. Shc adapter protein signaling complexes. J Biol Chem 1995;270:19969-19973.

26 Amin HM, McDonnell TJ, Ma Y, et al. Selective inhibition of STAT3 induces apoptosis and G(1) cell cycle arrest in ALK-positive anaplastic large cell lymphoma. Oncogene 2004;23:5426-5434.

27 Druker BJ. Imatinib as a paradigm of targeted therapies. Adv Cancer Res 2004;91:1-30.

28 Debelenko LV, Arthur DC, Pack SD, et al. Identification of CARS-ALK fusion in primary and metastatic lesions of an inflammatory myofibroblastic tumor. Lab Invest 2003;83:1255-1265.

29 Griffin CA, Hawkins AL, Dvorak C, et al. Recurrent involvement of 2p23 in inflammatory myofibroblastic tumors. Cancer Res 1999;59:2776-2780.

30 Bjornsti MA, Houghton PJ. The TOR pathway: a target for cancer therapy. Nat Rev Cancer 2004;4:335-348.

31 Atwell S, Adams JM, Badger J, et al. A novel mode of gleevec (STI-571, Imatinib) binding is revealed by the structure of spleen tyrosine kinase (Syk). J Biol Chem 2004;279:55827-55832.

32 O'Hare T, Pollock R, Stoffregen EP, et al. Inhibition of wild-type and mutant Bcr-Abl by AP23464, a potent ATP-based oncogenic protein kinase inhibitor: implications for CML. Blood 2004;104:2532-2539.

33 O'Shea JJ, Pesu M, Borie DC, et al. A new modality for immunosuppression: targeting the JAK/STAT pathway. Nat Rev Drug Discov 2004;3:555-564.

34 Crockett D, Lin KZ, Elenitoba-Johnson KS, et al. Identification of NPM-ALK interacting proteins by tandem mass spectrometry. Oncogene 2004;23:26172629 . 